\title{
Corrosion shape reconstruction of the mixed boundary in electrostatic imaging
}

\author{
Yuqing $\mathrm{Hu}^{1 *}\left(\mathbb{D}\right.$, Bingxian Wang ${ }^{2}$ and Tongxing $\mathrm{Li}^{3}$
}

\author{
"Correspondence: yqhu@jit.edu.cn \\ 'School of Science, Jinling Institute \\ of Technology, Hongjing Road 99, \\ Nanjing, 211169, P.R. China \\ Full list of author information is \\ available at the end of the article
}

\begin{abstract}
We consider the corrosion detection problem in terms of the Laplace equation and study a simply connected bounded domain with Wentzell-type GIBC boundary condition. We derive the systems of integral equations and establish the equivalence to the inverse shape problem in a Sobolev space setting. For the direct problem, we use potential theory to simulate the Neumann data from Dirichlet data on Dirichlet boundary. Then we propose a Newton iterative approach based on the boundary integral equations derived from Green's representation theorem. After describing the linearization and the iteration scheme for the inverse shape, we compute the Fréchet derivatives with respect to the unknowns. We conclude by presenting several numerical examples for shape reconstructions to show the validity of the proposed method.
\end{abstract}

MSC: 35R30; 65F22; 65R20; 65R32

Keywords: Generalized impedance boundary condition (GIBC); Nonlinear integral equation; Iterative solution; Numerics

\section{Introduction}

The Laplace equation is a special partial differential equation. Solving the boundary value problem of the Laplace equation is an important mathematical problem often encountered in the fields of electromagnetics, astronomy, thermodynamics, and hydrodynamics, because such equations describe the properties of physical objects such as electric, gravitational, and flow fields in the form of potential function. Many physical problems, for instance, thermal imaging, electrostatic imaging, and corrosion detection, are mathematically regarded as inverse boundary value problems of the Laplace equation. Such inverse problems can be interpreted as reconstructing the boundary shape or the impedance coefficients on the boundary from the measurements. Much work has been done on these problems $[7,10,11,13,14,21,22,28]$, where the authors used the nonlinear integral equation method proposed by Kress and Rundell [24]. In [10, 13, 14] the authors applied the method of [24] to reconstruct the impedance boundary shape of the corrosion problems. The integral equation method is also widely used $[8,9,12]$.

In many cases, complex scattering problems need to be considered, which usually are described as the scattering problems with absorbing boundary or multiple scatterers

(c) The Author(s) 2020. This article is licensed under a Creative Commons Attribution 4.0 International License, which permits use sharing, adaptation, distribution and reproduction in any medium or format, as long as you give appropriate credit to the original author(s) and the source, provide a link to the Creative Commons licence, and indicate if changes were made. The images or other third party material in this article are included in the article's Creative Commons licence, unless indicated otherwise in a credit line to the material. If material is not included in the article's Creative Commons licence and your intended use is not permitted by statutory regulation or exceeds the permitted use, you will need to obtain permission directly from the copyright holder. To view a copy of this licence, visit http://creativecommons.org/licenses/by/4.0/. 
$[15,19,29]$ (and references therein). A layer of medium on the surface of a scatterer can be used to protect the coated scatterer from external environment corrosion and also can be used to absorb the incident wave according to its own needs to reduce the intensity of scattering to achieve stealth purpose. These problems can be mathematically described by the Laplace equation. The boundary conditions of scatterers are sometimes assumed because of physical requirements or limitations. At this time, some boundary conditions satisfy the Dirichlet condition, and others satisfy the damped boundary conditions or other nonDirichlet boundary conditions, resulting in Wentzell boundary problems. The scattering model of partial boundary coatings satisfies different kinds of boundary conditions in different parts of the boundary. Because the boundary impedance coefficients essentially reflect the absorption of energy by the boundary. On one hand, the scattering model can be invisible to the target by appropriately adjusting the coefficients. On the other hand, the boundary impedance coefficients can be appropriately adjusted as the task of antistealth. Therefore an important study in this kind of inverse scattering problem is reconstructing the boundary shape or the boundary impedance coefficients from the measured data of the forward problem $[2-7,25]$, so as to effectively identify the target object. In [7] the authors studied the inverse problem for a mixed boundary value problem of the Laplace equation. They considered a doubly connected bounded domain with exterior Dirichlet boundary condition and interior generalized impedance boundary condition (GIBC). They derived an integral equation system and proved the equivalence to the original inverse problem of reconstructing the boundary shape and boundary impedance in a Sobolev space setting.

In this paper, we consider a Wentzell-type GIBC boundary condition of the Laplace equation. Two generalized impedance coefficients of the corrosion boundary are known. The shape of the GIBC part is reconstructed from a set of Cauchy data measured on the known boundary. The direct problem is computing Neumann data from the corresponding Dirichlet data on the known boundary by the jump relation of the single-layer potential. Then we use a set of Cauchy data obtained from the direct problem to reconstruct the corrosion boundary shape with generalized impedance condition based on Green's formula. The background of this formulation is that the generalized impedance coefficients are defined on the corresponding boundary, so these coefficients can also be regarded as two functions of the shape of the unknown boundary. This problem of reconstructing the geometry, which is also included in the crack problems [21], can be regarded as a simplified model with unknown generalized impedance boundary. The inverse problem to be solved by Green's formula is transformed into a set of nonlinear integral equations with respect to the boundaries. The Fréchet derivatives are computed to linearize the system, and then the ill-posed equations are solved by regularization to reconstruct the boundary shape. We propose an iterative numerical inversion scheme for recovering the geometry. Because the solution of the boundary value problem of the Laplace equation is singular at the boundary points of the mixed boundary conditions in a simply connected region, qualitative theoretical research needs to be carried out in a more general function space. The inverse problem in this paper is an application based on the nonlinear integral equations proposed in [24].

Our paper is organized as follows. In the next section, we formulate the direct and inverse problems with mixed boundary conditions. In Sect. 3, we obtain a set of boundary integral equations according to Green's formula inside the cavity and the boundary jump relations. Thus the inverse problem of reconstructing the generalized impedance 
boundary shape of the cavity is transformed into solving the nonlinear boundary integral equations. Then we propose the Newton method to reconstruct the boundary shape. Firstly, we obtain the Fréchet derivative of corrosion boundary $\Gamma_{c}$ and transform the original nonlinear integral equations into linear integral equations. In addition, we use the regularized Newton iterative method to solve the linear equations equivalent to the inverse problem. We end the paper with various numerical examples of reconstructing the generalized impedance boundary shape. We also discuss the validity of the regularized Newton method and its stability to data errors.

\section{The inverse problem with mixed boundary conditions}

In this paper, we consider the inverse corrosion problem of electrostatic imaging in bounded domain under certain boundary conditions, which is usually considered as the inverse boundary value problem of the Laplace equation with respect to electrostatic potential or temperature. More specifically, let $D \subset \mathbb{R}^{2}$ be a simply connected bounded domain with piecewise smooth boundary $\partial D$. We assume that the boundary is composed as $\partial D=\bar{\Gamma}_{m} \cup \bar{\Gamma}_{c}$ where $\Gamma_{m}$ and $\Gamma_{c}$ are two connected open disjoint portions of $\partial D$ of class $C^{2}$ without cusps at two intersection points. Moreover, $\Gamma_{m}$ is assumed to be nonempty with $\left|\Gamma_{m}\right|>0$. Denote by $v$ the unit outward normal to $\partial D$ defined almost everywhere. We assume that $\partial D$ is the boundary of the cross-section of a cylindrical partially coated perfectly conducting cavity where $\Gamma_{c}$ represents the portion coated by a conducting material. For the electrostatic imaging problem, the electrostatic potential $u$ can be given as the following boundary value problem: given a function $f \in H^{\frac{3}{2}}\left(\Gamma_{m}\right)$, consider the Laplace equation

$$
\Delta u=0 \quad \text { in } D
$$

with mixed boundary condition

$$
\frac{\partial u}{\partial v}-\operatorname{div}_{\Gamma_{c}}\left(\mu \operatorname{grad}_{\Gamma_{c}} u\right)+\lambda u=0 \quad \text { on } \Gamma_{c}
$$

and

$$
u=f \quad \text { on } \Gamma_{m} \text {, }
$$

where $\lambda \in L^{\infty}\left(\Gamma_{c}\right)$ is not negative and nonconstant to zero, and $\mu \in L^{\infty}\left(\Gamma_{c}\right)$ is positive. Both $\lambda$ and $\mu$ denote the surface corrosion coefficients on the boundary $\Gamma_{c}$, $\operatorname{div}_{\Gamma_{c}}$ and $\operatorname{grad}_{\Gamma_{c}}$ are the divergence and gradient of the unknown boundary, respectively, and $f$ is the voltage on the boundary $\Gamma_{m}$.

Thus the forward problem is as follows: compute the corresponding Neumann data from Dirichlet data $f$ on $\Gamma_{m}$, which is the partial boundary of conductive medium. We define Neumann data

$$
g:=\frac{\partial u}{\partial v} \quad \text { on } \Gamma_{m}
$$

For the following discussion, we define the data $(f, g) \in H^{\frac{3}{2}}\left(\Gamma_{m}\right) \times H^{\frac{1}{2}}\left(\Gamma_{m}\right)$ on the boundary $\Gamma_{m}$ as Cauchy data such that $(f, g) \neq 0$. In the two-dimensional case the inhomogeneous 
Laplace-Beltrami differential operator $\operatorname{div}_{\Gamma_{c}}\left(\mu \operatorname{grad}_{\Gamma_{c}} u\right)$ can be rewritten as [20]

$$
\operatorname{div}_{\Gamma_{c}}\left(\mu \operatorname{grad}_{\Gamma_{c}} u\right)=\frac{d}{d s} \mu \frac{d u}{d s}
$$

where $d / d s$ is the tangent derivative, and $s$ is the arc length of $\Gamma_{c}$. The latter is the form we use from now on.

In particular, given $f \in H^{\frac{3}{2}}\left(\Gamma_{m}\right)$, there exists a unique harmonic function $u \in H^{2}(D)$ satisfying the generalized impedance condition (2) on $\Gamma_{c}$ and the Dirichlet boundary condition $u=f$ on $\Gamma_{m}$. This is a boundary value problem: For $f \in H^{\frac{3}{2}}\left(\Gamma_{m}\right)$ and $h \in H^{\frac{1}{2}}\left(\Gamma_{c}\right)$, find a solution $u \in H^{2}(D)$ such that

$$
\begin{aligned}
& \Delta u=0 \quad \text { in } D, \\
& u=f \quad \text { on } \Gamma_{m},
\end{aligned}
$$

and

$$
\frac{\partial u}{\partial v}-\operatorname{div}_{\Gamma_{c}}\left(\mu \operatorname{grad}_{\Gamma_{c}} u\right)+\lambda u=h \quad \text { on } \Gamma_{c},
$$

where $\lambda \in L^{\infty}\left(\Gamma_{c}\right)$ is nonnegative and not identically zero, $\mu \in L^{\infty}\left(\Gamma_{c}\right)$ is positive, and $s$ is the arc length variable on $\Gamma_{c}$. Suppose $f=0$ and $h=0$. Applying Green's first integral theorem and using the boundary condition, we have that $[3,11]$

$$
\int_{D}|\nabla u|^{2} d x=\int_{D} u \frac{\partial u}{\partial v} d s=-\int_{\Gamma_{c}} \mu\left|\frac{d u}{d s}\right|^{2} d s-\int_{\Gamma_{c}} \lambda|u|^{2} d s
$$

In view of the positivity of $\lambda$ and $\mu$, we have $u=0$ in $D$, which yields that the boundary value problem (5)-(7) has at most one solution. Hence, for $f \in H^{\frac{3}{2}}\left(\Gamma_{m}\right)$, there exists a unique solution $u \in H^{2}(D)$ of (1)-(3).

The inverse problem is as follows: determine the shape of the corrosion boundary $\Gamma_{c}$ from Cauchy data on $\Gamma_{m}$.

Bacchelli [1] discussed the impedance boundary on $\Gamma_{c}$, that is, $\mu=0$ in (2). She established that two pairs of Cauchy data on $\Gamma_{m}$ can uniquely determine both the shape of the domain $D$ and the impedance function $\lambda$ on $\partial D$, provided that $f_{1}$ and $f_{2}$ are linearly independent and one of them is positive. In [10] the authors considered the particular case of Neumann boundary data on $\Gamma_{c}, \lambda=0$ and $\mu=0$ in (2), and showed that one pair of Cauchy data can uniquely determine the missing part of the boundary. For our inverse problem, the uniqueness of the boundary $\Gamma_{c}$ with finitely many Cauchy pairs is an open problem, even if we assume that $\lambda$ and $\mu$ are known. We will not deal with identifiability result in this paper even although this is a crucial question.

\section{Integral equations}

Based on Green's representation formula, in this section, we derive two nonlinear integral equations equivalent to the inverse problem to be solved. To get nonlinear integral equations from the fundamental solution of the Laplace equation in $\mathbb{R}^{2}$

$$
\Phi(x, y)=\frac{1}{2 \pi} \ln \frac{1}{|x-y|}, \quad x \neq y
$$


we define the single potential operator $S: H^{-\frac{1}{2}}(\partial D) \rightarrow H^{\frac{1}{2}}(\partial D)$ by

$$
(S \phi)(x):=\int_{\partial D} \Phi(x, y) \phi(y) d s(y), \quad x \in \partial D
$$

and the double potential operator $K: H^{\frac{1}{2}}(\partial D) \rightarrow H^{\frac{1}{2}}(\partial D)$ by

$$
(K \phi)(x):=\int_{\partial D} \frac{\partial \Phi(x, y)}{\partial v(y)} \phi(y) d s(y), \quad x \in \partial D .
$$

The forms of the operators on the boundaries are

$$
\left(S_{j k} \phi\right)(x):=\int_{\Gamma_{j}} \Phi(x, y) \phi(y) d s(y), \quad x \in \Gamma_{k},
$$

and

$$
\left(K_{j k} \phi\right)(x):=\int_{\Gamma_{j}} \frac{\partial \Phi(x, y)}{\partial v(y)} \phi(y) d s(y), \quad x \in \Gamma_{k},
$$

respectively, where $j, k=m, c$.

From now on, without loss of generality, we assume that there exists a point $x^{*} \in D$ such that $\left|x-x^{*}\right| \neq 1$ for all $x \in \partial D$. Then by the definition of a single potential operator in (8) the operator $S$ is injective [23]. Suppose that $u \in H^{2}(D)$ is the solution to the boundary value problem (1)-(3). To simplify the definition, let

$$
\eta:=\left.u\right|_{\Gamma_{c}}
$$

and then denote

$$
\Psi:=\frac{d}{d s} \mu \frac{d \eta}{d s}-\lambda \eta
$$

Using Green's formula, two different boundary conditions, and the Cauchy data on the boundaries, we derive

$$
\begin{aligned}
u(x)= & \int_{\Gamma_{m}}\left\{\Phi(x, y) g(y)-\frac{\partial \Phi(x, y)}{\partial v(y)} f(y)\right\} d s(y) \\
& +\int_{\Gamma_{c}}\left\{\Phi(x, y) \Psi(y)-\frac{\partial \Phi(x, y)}{\partial v(y)} \eta(y)\right\} d s(y), \quad x \in D .
\end{aligned}
$$

Let $x$ be close to the two boundaries $\Gamma_{m}$ and $\Gamma_{c}$ from the inside of the cavity $D$ based on (13). By means of the jump relations of double potential and the continuity of the single potential on the boundary, we get the following nonlinear boundary integral equations:

$$
\begin{aligned}
& S_{m m} g+S_{c m}\left(\frac{d}{d s} \mu \frac{d \eta}{d s}-\lambda \eta\right)-K_{c m} \eta=\frac{f}{2}+K_{m m} f, \quad x \in \Gamma_{m}, \\
& S_{m c} g+S_{c c}\left(\frac{d}{d s} \mu \frac{d \eta}{d s}-\lambda \eta\right)-K_{c c} \eta-\frac{\eta}{2}=K_{m c} f, \quad x \in \Gamma_{c},
\end{aligned}
$$


where $g:=\left.\frac{\partial u}{\partial \nu}\right|_{\Gamma_{m}} \in H^{\frac{1}{2}}\left(\Gamma_{m}\right), \eta \in H^{\frac{3}{2}}\left(\Gamma_{c}\right)$, and $m, c$ denote the corresponding two different boundaries.

Conversely, assume that given a Cauchy pair $(f, g)$ on $\Gamma_{m}$, the functions $\lambda$ and $\mu$ on $\Gamma_{c}$, the curve $\Gamma_{c}$, and $\eta$ solve the system of integral equations (14) and (15), define the function $u$ by the right-hand side of (13) for all $x \in R^{2} \backslash \partial D$. Then $\Delta u=0$ in $D$ and $R^{2} \backslash \bar{D}$. From the integral equations (14) and (15) and the jump relations it follows that the limits of $u$ obtained by approaching $\partial D$ from outside vanish. The uniqueness for the exterior Dirichlet problem, together with some consideration on the behavior of the single-layer potentials at infinity using our assumption that there exists $x^{*} \in D$ such that $\left|x-x^{*}\right| \neq 1$ for all $x \in \partial D$, now implies that $u$ vanishes in $R^{2} \backslash \bar{D}$. Again, the jump relations finally yield that the harmonic function $u$ has Cauchy data $(f, g)$ on $\Gamma_{m}$ and Dirichlet values $u=\eta$ on $\Gamma_{c}$ and satisfies the generalized impedance condition with coefficients $\lambda$ and $\mu$ on $\Gamma_{c}$. Hence we can state the following equivalence (see also $[13,14]$ ).

Theorem 3.1 Reconstructing the shape of corrosion boundary is equivalent to finding the solution $\Gamma_{c}$ and $\eta$ from equations (14)-(15).

Theorem 3.2 For given impedance coefficients $\lambda$ and $\mu$, the integral equation (15) has a unique solution $\eta \in H^{\frac{3}{2}}\left(\Gamma_{c}\right)$ [11].

More specifically, equations (14) and (15) are linear with respect to $\eta$, but they are nonlinear with respect to the reconstructed boundary $\Gamma_{c}$. Equation (15) is well posed, whereas the data equation (14) is seriously ill-posed for the inverse problem. Suppose that we can measure accurate or slightly perturbed Cauchy data; however, because of the ill-poseness of the equation, a stable regularization method is needed to solve equation (14) in the process of inverse problem. Because the $L^{2}$-norm is often used to measure the error data, considering equation (14) under the $L^{2}$-norm is better than discussing the equation in its trace space.

To solve the nonlinear integral equations (14)-(15), we adopt the Newton iterative method. We solve equations (14)-(15) simultaneously to get the solutions $\Gamma_{c}$ and $\eta$ by iteration. This means that we need to linearize equations (14) and (15) simultaneously corresponding to the two unknowns $\Gamma_{c}$ and $\eta$, which are required to be solved.

\section{The iterative method of the inverse problem}

In this section, we present a Newton iterative method for solving nonlinear boundary integral equations (14)-(15). Based on the nonlinearity of equations (14)-(15) on the boundary $\Gamma_{c}$, we need to linearize equations (14)-(15) respect to the corrosion boundary curve $\Gamma_{c}$ by solving the Fréchet derivatives of the integral operators on the boundary. Thus the unknowns of the inverse problem can be solved in the linear systems of the equations. First, we parameterize the boundary and its integral operators.

\subsection{Integral equations parameterization}

For further investigation of the integral equations, we present

$$
\partial D=\{z(t): t \in[0,2 \pi]\}
$$


with a $2 \pi$-periodic $C^{2}$-smooth function $z: \mathbb{R} \rightarrow \mathbb{R}^{2}$ such that $z$ is injective on $[0,2 \pi)$ and satisfies $z^{\prime}(t) \neq 0$ for all $t$. Without losing generality, suppose that

$$
\Gamma_{m}=\left\{z_{m}(t): t \in(0, \pi)\right\}, \quad \Gamma_{c}=\left\{z_{c}(t): t \in(\pi, 2 \pi)\right\}
$$

are the parameterized representations of $C^{2}$-boundaries $\Gamma_{m}$ and $\Gamma_{c}$, respectively. To incorporate possible singularities of the solutions at the end points of $\Gamma_{c}$ and $\Gamma_{m}$, we can employ sigmoidal transformations; see $[17,18]$. Here we write $a^{\perp}=\left(a_{2},-a_{1}\right)$ for any vector $a=\left(a_{1}, a_{2}\right)$, that is, $a^{\perp}$ is obtained by rotating $a$ clockwise by $90^{\circ}$. For $j, k=m, c$, denote $\psi=\phi \circ z_{j}$. In view of (10) and (11), we introduce the parameterized single- and doublelayer operators $\tilde{S}_{j k}: H^{-\frac{1}{2}}(\partial D) \rightarrow H^{\frac{1}{2}}(\partial D)$ and $\tilde{K}_{j k}: H^{\frac{1}{2}}(\partial D) \rightarrow H^{\frac{1}{2}}(\partial D)$ by

$$
\left(\tilde{S}_{c j} \psi\right)(x):=\frac{1}{2 \pi} \int_{0}^{\pi} \ln \frac{1}{\left|z_{j}(t)-z_{c}(\tau)\right|}\left|z_{c}^{\prime}(\tau)\right| \psi(\tau) d \tau
$$

and

$$
\left(\tilde{K}_{c j} \psi\right)(x):=\frac{1}{2 \pi} \int_{0}^{\pi} \frac{\left[z_{c}^{\prime}(\tau)\right]^{\perp} \cdot\left[z_{j}(t)-z_{c}(\tau)\right]}{\left|z_{j}(t)-z_{c}(\tau)\right|^{2}} \psi(\tau) d \tau
$$

To discretize the integral operators, the kernel of the operator $\tilde{S}_{j j}$ can be decomposed as

$$
\ln \frac{1}{\left|z_{j}(t)-z_{j}(\tau)\right|}=-\ln \left|\sin \frac{t-\tau}{2}\right|+\ln \frac{\left|\sin \frac{t-\tau}{2}\right|}{\left|z_{j}(t)-z_{j}(\tau)\right|}, \quad j=c, m,
$$

where the second item is smooth, and the diagonal value is

$$
\lim _{\tau \rightarrow t} \ln \frac{\left|\sin \frac{t-\tau}{2}\right|}{\left|z_{j}(t)-z_{j}(\tau)\right|}=-\ln 2\left|z_{j}^{\prime}(t)\right|
$$

The kernel of the operator $\tilde{K}_{j k}$ is smooth; when $j=k$, the element on diagonals becomes

$$
\lim _{\tau \rightarrow t} \frac{\left[z_{j}^{\prime}(\tau)\right]^{\perp} \cdot\left[z_{j}(t)-z_{j}(\tau)\right]}{\left|z_{j}(t)-z_{j}(\tau)\right|^{2}}=\frac{\left[z_{j}^{\prime}(t)\right]^{\perp} \cdot z_{j}^{\prime \prime}(t)}{2\left|z_{j}^{\prime}(t)\right|^{2}}, \quad j=c, m .
$$

\subsection{Newton iterative method and computation of Fréchet derivatives}

Now we present an algorithm for solving the linear integral equations (14)-(15), the Newton Iterative Method. Linearizing equations (14)-(15) completely corresponding to the unknown boundary shape $\Gamma_{c}$ and the boundary value $\eta$, we get the equations

$$
\begin{aligned}
\tilde{S}_{c c}( & \left.\frac{d}{d t} \mu \frac{d \eta}{d t}-\lambda \eta, z_{c}\right)+\tilde{S}_{c c}\left(\frac{d}{d t} \mu \frac{d \gamma}{d t}-\lambda \gamma, z_{c}\right)+d \tilde{S}_{c c}\left[\Psi, z_{c} ; \zeta\right] \\
& -\tilde{K}_{c c}\left(\eta, z_{c}\right)-\tilde{K}_{c c}\left(\gamma, z_{c}\right)-d \tilde{K}_{c c}\left[\eta, z_{c} ; \zeta\right]-\frac{\eta}{2}-\frac{\gamma}{2} \\
= & w_{c}+\zeta \cdot\left(\operatorname{grad}\left(w_{c}\right)\right) \circ z_{c}, \quad x \in \Gamma_{c},
\end{aligned}
$$

and

$$
\tilde{S}_{c m}\left(\frac{d}{d t} \mu \frac{d \eta}{d t}-\lambda \eta, z_{c}\right)+\tilde{S}_{c m}\left(\frac{d}{d t} \mu \frac{d \gamma}{d t}-\lambda \gamma, z_{c}\right)+d \tilde{S}_{c m}\left[\Psi, z_{c} ; \zeta\right]
$$




$$
-\tilde{K}_{c m}\left(\eta, z_{c}\right)-\tilde{K}_{c m}\left(\gamma, z_{c}\right)-d \tilde{K}_{c m}\left[\eta, z_{c} ; \zeta\right]=w_{m}, \quad x \in \Gamma_{m}
$$

where $w_{c}:=\tilde{K}_{m c} f-\tilde{S}_{m c} g, w_{m}:=\frac{f}{2}+\tilde{K}_{m m} f-\tilde{S}_{m m} g$, the operators $d \tilde{S}_{c m}\left[\Psi, z_{c} ; \zeta\right], d \tilde{K}_{c m}\left[\eta, z_{c} ; \zeta\right]$, $d \tilde{S}_{c c}\left[\Psi, z_{c} ; \zeta\right]$, and $d \tilde{K}_{c c}\left[\eta, z_{c} ; \zeta\right]$ represent the Fréchet derivatives of $\tilde{S}_{c m} \Psi, \tilde{K}_{c m} \eta, \tilde{S}_{c c} \Psi$, and $\tilde{K}_{c c} \eta$, along the direction of $\zeta$ with respect to the unknown boundary $z_{c}$.

The method can be summarized as three steps:

1. Given the initial value of $z_{c}$, which is the parameterized form of corrosion boundary $\Gamma_{c}$. Compute the solution from the well-posed equation (15), given as the initial value of $\eta$.

2. Plug the initial values of $z_{c}$ and $\eta$ obtained in the first step into equations (20)-(21). Solve the linearized system (20)-(21) by Tikhonov regularization for $\zeta$ and $\gamma$. Hence the unknown boundary is updated as $z_{c}+\zeta$, whereas the boundary value is updated as $\eta+\gamma$.

3. Repeat the second step until the iteration stop criterion is satisfied.

Remark 4.1 In addition to the Newton iterative method for solving the inverse problems of (14) and (15), there are also two feasible methods. From the ill-posed equation (14) we get the density $\eta$, then linearize the well-posed equation (15), so the approximate boundary can be obtained by iterative updating. This method is referred in [10, 27]. Similarly, compute $\eta$ from the well-posed equation (15), then linearize the ill-posed equation(14), and reconstruct the corrosion boundary shape by regularization method.

We use the Tikhonov regularization method in the second step of iteration. Without regularization, the equations are unstable, that is, strong numerical oscillations appear. More specifically, we add the penalty terms

$$
\alpha_{\zeta}\|\zeta\|_{H^{2}}^{2}+\alpha_{\gamma}\|\gamma\|_{L^{2}}^{2}
$$

to the square of the $L_{2}$-norm of the difference of the left- and right-hand sides of equations (20)-(21) as usual in the Tikhonov regularization, where $\alpha_{\zeta}, \alpha_{\gamma}$ are the regularization parameters to the respective unknowns. Penalizing not only the function values but also the first and second derivatives adds stability. For this reason, we choose the $H^{2}$ penalty term to the reconstructed boundary $\zeta$ due to its nonlinearity and the $L^{2}$ penalty term to the density $\eta$. We use Tikhonov regularization to solve the equations and use the projection method to solve the inverse problem.

To simplify the computation, we present some operators. The Fréchet derivatives of the operators $\tilde{S}_{j k}$ and $\tilde{K}_{j k}$ can be derived from the differential of the smooth kernel of the operators with respect to the unknown $z_{c}$ [26]. Thus, the Fréchet derivatives of the operators $\tilde{S}_{c m}$ and $\tilde{K}_{c m}$ can be computed as follows:

$$
\begin{aligned}
d \tilde{S}_{c m}\left[\Psi, z_{c} ; \zeta\right](t)= & \frac{1}{2 \pi} \int_{0}^{\pi} \frac{\left[z_{m}(t)-z_{c}(\tau)\right] \cdot \zeta(\tau)}{\left|z_{m}(t)-z_{c}(\tau)\right|^{2}} \frac{d}{d \tau} \frac{\mu}{\left|z_{c}^{\prime}(\tau)\right|} \frac{d \eta}{d \tau} d \tau \\
& +\frac{1}{2 \pi} \int_{0}^{\pi} \ln \frac{1}{\left|z_{m}(t)-z_{c}(\tau)\right|} \frac{d}{d \tau} \mu\left(-\frac{1}{\left|z_{c}^{\prime}(\tau)\right|^{2}} \frac{z_{c}^{\prime}(\tau) \cdot \zeta^{\prime}(\tau)}{\left|z_{c}^{\prime}(\tau)\right|}\right) \frac{d \eta}{d \tau} d \tau \\
& -\frac{1}{2 \pi} \int_{0}^{\pi} \frac{\left[z_{m}(t)-z_{c}(\tau)\right] \cdot \zeta(\tau)}{\left|z_{m}(t)-z_{c}(\tau)\right|^{2}}\left|z_{c}^{\prime}(\tau)\right| \lambda \eta d \tau
\end{aligned}
$$




$$
\begin{aligned}
& -\frac{1}{2 \pi} \int_{0}^{\pi} \ln \frac{1}{\left|z_{m}(t)-z_{c}(\tau)\right|} \lambda \eta \frac{z_{c}^{\prime}(\tau) \cdot \zeta^{\prime}(\tau)}{\left|z_{c}^{\prime}(\tau)\right|} d \tau, \\
d \tilde{K}_{c m}\left[\psi, z_{c} ; \zeta\right](t)= & \frac{1}{2 \pi} \int_{0}^{\pi} \frac{\left[\zeta^{\prime}(\tau)\right]^{\perp} \cdot\left[z_{m}(t)-z_{c}(\tau)\right]-\left[z_{c}^{\prime}(\tau)\right]^{\perp} \cdot \zeta(\tau)}{\left|z_{m}(t)-z_{c}(\tau)\right|^{2}} \psi(\tau) d \tau \\
& +\frac{1}{\pi} \int_{0}^{\pi} \frac{\left[z_{c}^{\prime}(\tau)\right]^{\perp} \cdot\left[z_{m}(t)-z_{c}(\tau)\right]\left[z_{m}(t)-z_{c}(\tau)\right] \cdot \zeta(\tau)}{\left|z_{m}(t)-z_{c}(\tau)\right|^{4}} \psi(\tau) d \tau,
\end{aligned}
$$

where $t \in[0, \pi]$. Similarly, we get

$$
\begin{aligned}
d \tilde{S}_{c c}\left[\Psi, z_{c} ; \zeta\right](t)= & \frac{1}{2 \pi} \int_{0}^{\pi} \frac{\left[z_{c}(t)-z_{c}(\tau)\right] \cdot[\zeta(t)-\zeta(\tau)]}{\left|z_{c}(t)-z_{c}(\tau)\right|^{2}} \frac{d}{d \tau} \frac{\mu}{\left|z_{c}^{\prime}(\tau)\right|} \frac{d \eta}{d \tau} d \tau \\
& -\frac{1}{2 \pi} \int_{0}^{\pi} \ln \frac{1}{\left|z_{c}(t)-z_{c}(\tau)\right|} \frac{d}{d \tau} \mu\left(-\frac{1}{\left|z_{c}^{\prime}(\tau)\right|^{2}} \frac{z_{c}^{\prime}(\tau) \cdot \zeta^{\prime}(\tau)}{\left|z_{c}^{\prime}(\tau)\right|}\right) \frac{d \eta}{d \tau} d \tau \\
& +\frac{1}{2 \pi} \int_{0}^{\pi} \frac{\left[z_{c}(t)-z_{c}(\tau)\right] \cdot[\zeta(t)-\zeta(\tau)]}{\left|z_{c}(t)-z_{c}(\tau)\right|^{2}}\left|z_{c}^{\prime}(\tau)\right| \lambda \eta d \tau \\
& -\frac{1}{2 \pi} \int_{0}^{\pi} \ln \frac{1}{\left|z_{c}(t)-z_{c}(\tau)\right|} \lambda \eta \frac{z_{c}^{\prime}(\tau) \cdot \zeta^{\prime}(\tau)}{\left|z_{c}^{\prime}(\tau)\right|} d \tau,
\end{aligned}
$$

where $t \in[\pi, 2 \pi]$. The kernel of the operator $d \tilde{S}_{c c}$ is smooth, and the diagonal values of the first and third items can be shown as

$$
\lim _{\tau \rightarrow t} \frac{\left[z_{c}(t)-z_{c}(\tau)\right] \cdot[\zeta(t)-\zeta(\tau)]}{\left|z_{c}(t)-z_{c}(\tau)\right|^{2}}=\frac{z_{c}^{\prime}(t) \cdot \zeta^{\prime}(t)}{\left|z_{c}^{\prime}(t)\right|^{2}} .
$$

The Fréchet derivative of the operator $\tilde{K}_{c c}$ along the direction $\zeta$ can be computed as

$$
\begin{aligned}
d \tilde{K}_{c c}\left[\psi, z_{c} ; \zeta\right](t)= & \frac{1}{\pi} \int_{0}^{\pi}\left(\frac{\left[z_{c}^{\prime}(\tau)\right]^{\perp} \cdot[\zeta(t)-\zeta(\tau)]+\left[\zeta^{\prime}(\tau)\right]^{\perp} \cdot\left[z_{c}(t)-z_{c}(\tau)\right]}{2\left|z_{c}(t)-z_{c}(\tau)\right|^{2}} \psi(\tau)\right. \\
& \left.-\frac{\left[z_{c}^{\prime}(\tau)\right]^{\perp} \cdot\left[z_{c}(t)-z_{c}(\tau)\right]\left[z_{c}(t)-z_{c}(\tau)\right] \cdot[\zeta(t)-\zeta(\tau)]}{\left|z_{c}(t)-z_{c}(\tau)\right|^{4}} \psi(\tau)\right) d \tau,
\end{aligned}
$$

where $t \in[\pi, 2 \pi]$. The kernel $K(t, \tau)$ of the operator $d \tilde{K}_{c c}$ is smooth with the diagonal value

$$
\lim _{\tau \rightarrow t} K(t, \tau)=\frac{\left[z_{c}^{\prime}(t)\right]^{\perp} \cdot \zeta^{\prime \prime}(t)+\left[\zeta^{\prime}(t)\right]^{\perp} \cdot z_{c}^{\prime \prime}(t)}{4\left|z_{c}^{\prime}(t)\right|^{2}}-\frac{\left[z_{c}^{\prime}(t)\right]^{\perp} \cdot z_{c}^{\prime \prime}(t) z_{c}^{\prime}(t) \cdot \zeta^{\prime}(t)}{2\left|z_{c}^{\prime}(t)\right|^{4}} .
$$

Let $w_{c}=w \circ z_{c}$ be the parametric form of the single- and double-layer potential of the unknown boundary $\Gamma_{c}$. The Frćchet derivative of the potential $w_{c}$ along the direction $\zeta$ with respect to the unknown boundary $z_{c}$ can be expressed as,

$$
\begin{aligned}
& \zeta \cdot\left(\operatorname{grad}\left(w_{c}\right)\right) \circ z_{c} \\
&=\frac{1}{2 \pi} \int_{\pi}^{2 \pi} f\left(z_{m}(\tau)\right) \frac{\left[z_{m}^{\prime}(\tau)\right]^{\perp} \cdot \zeta(t)}{\left|z_{c}(t)-z_{m}(\tau)\right|^{2}} d \tau \\
&-\frac{1}{\pi} \int_{\pi}^{2 \pi} f\left(z_{m}(\tau)\right) \frac{\left[z_{m}^{\prime}(\tau)\right]^{\perp} \cdot\left[z_{c}(t)-z_{m}(\tau)\right]\left[z_{c}(t)-z_{m}(\tau)\right] \cdot \zeta(t)}{\left|z_{c}(t)-z_{m}(\tau)\right|^{4}} d \tau \\
&+\frac{1}{2 \pi} \int_{\pi}^{2 \pi} g\left(z_{m}(\tau)\right) \frac{\left[z_{c}(t)-z_{m}(\tau)\right] \cdot \zeta(t)}{\left|z_{c}(t)-z_{m}(\tau)\right|^{2}}\left|z_{m}^{\prime}(\tau)\right| d \tau, \quad \text { where } t \in[\pi, 2 \pi] .
\end{aligned}
$$




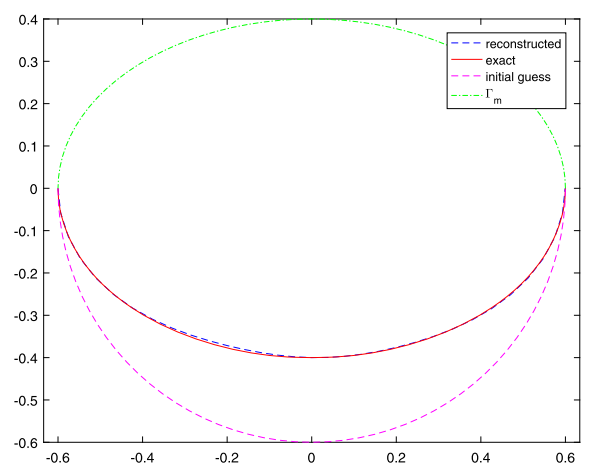

(a) Using exact Cauchy data, 7 iteration steps

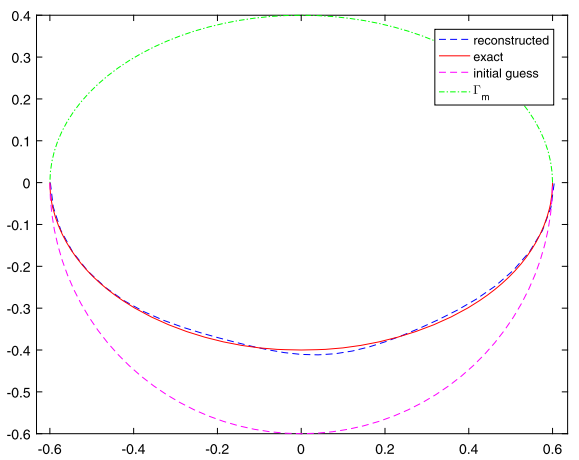

(b) Using noisy data with $\delta=5 \%, 9$ iteration steps

Figure 1 Reconstruction of the corrosion boundary of ellipse with exact data above and with $5 \%$ noisy data below, respectively. We choose Dirichlet data on $\Gamma_{m}$ as $f\left(z_{m}(t)\right)=1, t \in[0, \pi]$, the impedance coefficients on boundary $\Gamma_{C}$ are chosen as $\lambda=0.8+\cos ^{4} t$ and $\mu=\frac{1}{1+0.15 \cos 2 t}, t \in[\pi, 2 \pi]$

\section{Numerical examples}

In this section, we give some numerical examples to verify the feasibility of the proposed Newton iterative method. We solve the solution to the direct problem by the single-layer potential method [11]. We use the Green's formula method to reconstruct the corrosion boundary curve. However, because of singularities of the solution to (1)-(3) at the two intersection points, discretizing the equations with equidistant points on $[0,2 \pi]$ would lead to a poor accuracy. For this reason, it is more appropriate to use a mesh graded toward the intersection points $[13,14,16]$. Such a grading can be achieved most efficiently by using a sigmoidal transformation. We parameterize the unknown part of the boundary in the form that automatically satisfies the continuity condition to overcome a possible mismatch between two boundaries. Note that the boundary integral equations for creating the data are obtained via the potential approach, whereas the integral equations in the inverse algorithm are based on Green's formula, and thus committing an inverse crime is avoided. 


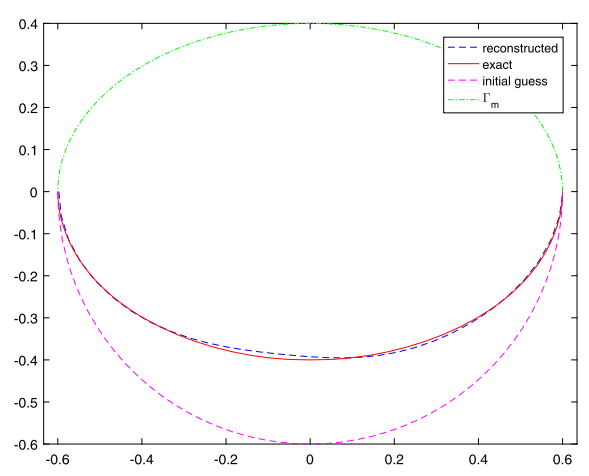

(a) Using exact Cauchy data, 13 iteration steps

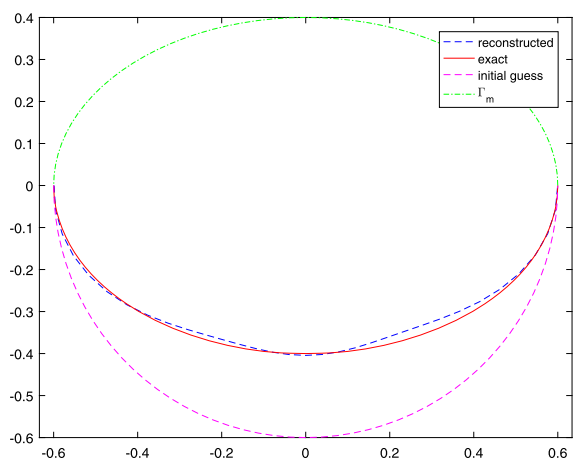

(b) Using noisy data with $\delta=3 \%, 13$ iteration steps

Figure 2 Reconstruction the corrosion boundary of ellipse with exact data above and with 3\% noisy data below, respectively. We choose Dirichlet data on $\Gamma_{m}$ as $f\left(z_{m}(t)\right)=1+\cos ^{2}(t), t \in[0, \pi]$, the impedance coefficients on boundary $\Gamma_{c}$ are chosen as $\lambda=0.8+\cos ^{4} t$ and $\mu=\frac{1}{1+0.15 \cos 2 t}, t \in[\pi, 2 \pi]$

In principle, the parameterization of the update $\zeta$ obtained from (20) and (21) is not unique. To cope with this ambiguity, we use star-like parameterizations of the form

$$
z_{c}(t)=r(t)(\cos t, \sin t), \quad t \in[\pi, 2 \pi]
$$

with a nonnegative function $r$ representing the radial distance of $\Gamma_{c}$ from the origin. Consequently, the perturbations are of the form

$$
\zeta(t)=q(t)(\cos t, \sin t), \quad t \in[\pi, 2 \pi]
$$

with a real function $q$. In the process of iteration approximation, we assume that $q$ can be extended to a trigonometric polynomial form which the series less than or equal to $m \in \mathbb{N}$, more specifically,

$$
q(t) \approx \sum_{j=0}^{m} a_{j} \cos (j t)+\sum_{j=1}^{m} b_{j} \sin (j t) .
$$




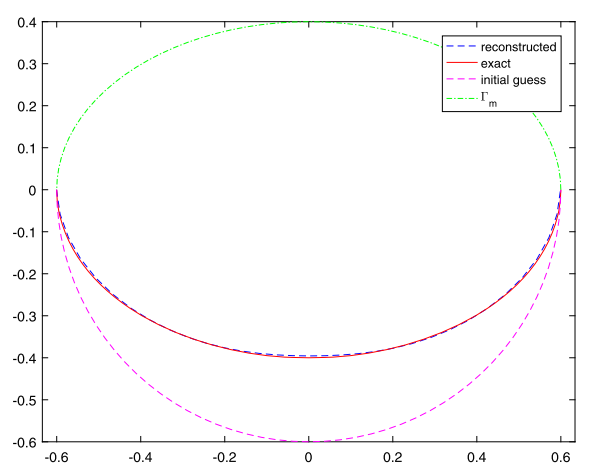

(a) Using exact Cauchy data, 12 iteration steps

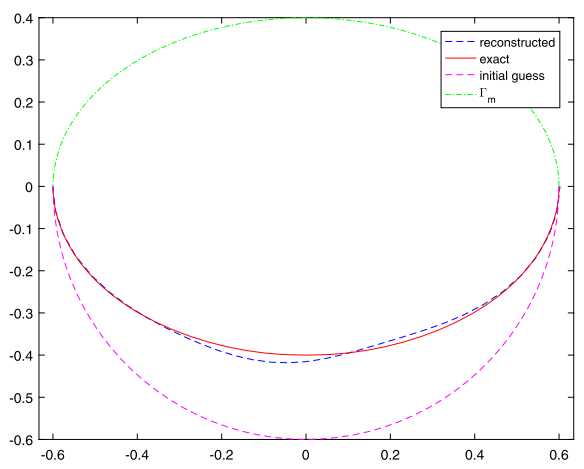

(b) Using noisy data with $\delta=3 \%, 13$ iteration steps

Figure 3 Reconstruction the corrosion boundary of ellipse with exact data above and with 3\% noisy data below, respectively. We choose Dirichlet data on $\Gamma_{m}$ as $f\left(z_{m}(t)\right)=1+\cos ^{2}(t), t \in[0, \pi]$, the impedance coefficients on boundary $\Gamma_{c}$ are chosen as two constants $\lambda=1$ and $\mu=3$

We choose 64 configuration points on the two mixed boundaries in the simply connected region, that is, $m=32$. For the perturbed data, the random error with $L^{2}$-norm is

$$
g^{\delta}:=g+\delta \frac{\|g\|_{L^{2}}}{\|\rho\|_{L^{2}}} \rho
$$

where $g$ is the exact data, $\delta$ is the relative error level, and $\rho$ is a random variable of normal distribution. The feasibility of the algorithm is verified by the initial values of different impedance coefficients in numerical examples. The following examples solve equations (20) and (21) approximately by using Tikhonov regularization. In the process of regularization, we choose the $H^{2}$-penalty term to the reconstructed boundary $\zeta$. For the density function $\eta$, we choose the $L^{2}$-penalty term. We use the projection method to solve the inverse problem. Of course, based on the Newton iterative method mentioned previously, an appropriate algorithm stopping criterion is needed to terminate the operation. In our numerical examples, our iteration steps and regularization parameters are chosen 


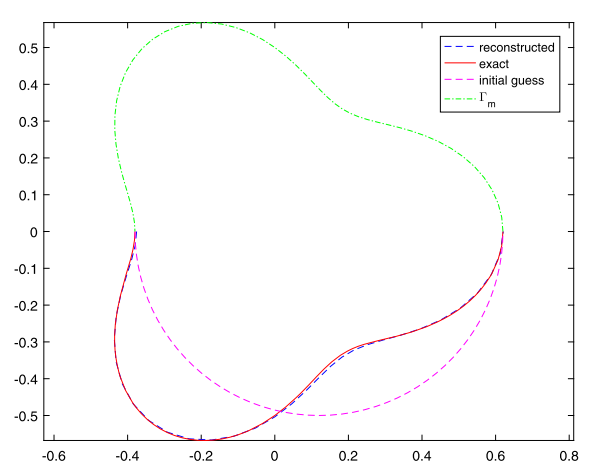

(a) Using exact Cauchy data, 7 iteration steps

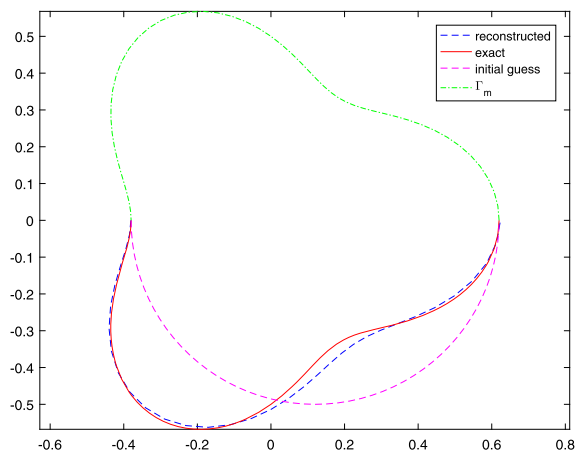

(b) Using noisy data with $\delta=2 \%, 9$ iteration steps

Figure 4 Reconstruction the corrosion boundary of pear with exact data above and with $2 \%$ noisy data below, respectively. We choose Dirichlet data on $\Gamma_{m}$ as $f\left(z_{m}(t)\right)=1, t \in[0, \pi]$, the impedance coefficients on boundary $\Gamma_{c}$ are chosen as $\lambda=0.8+\cos ^{4} t$ and $\mu=\frac{1}{1+0.15 \cos 2 t}, t \in[\pi, 2 \pi]$

experimentally. In the first two numerical examples, we choose different Dirichlet data and different impedance coefficients to show the validity of our method.

Example 1 We consider the parametric form of elliptic boundary

$$
z_{c}(t)=(0.6 \cos t, 0.4 \sin t), \quad t \in[\pi, 2 \pi]
$$

and choose $m=5$ in (24) and the given parametric form of known boundary $\Gamma_{m}$

$$
z_{m}(t)=(0.6 \cos t, 0.4 \sin t), \quad t \in[0, \pi]
$$

We suppose $z_{c_{0}}(t)=(0.6 \cos t, 0.6 \sin t), t \in[\pi, 2 \pi]$, as the initial value of the boundary $\Gamma_{c}$. Choosing different Dirichlet data and different boundary impedance coefficients, the reconstruction method is feasible. In Fig. 1, we choose the Dirichlet data on $\Gamma_{m}$ as 


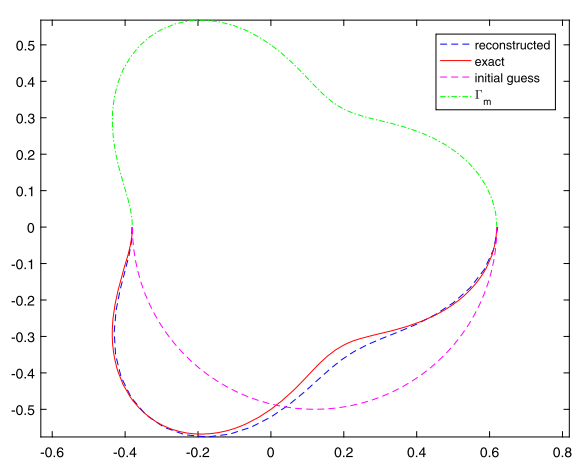

(a) Using exact Cauchy data, 7 iteration steps

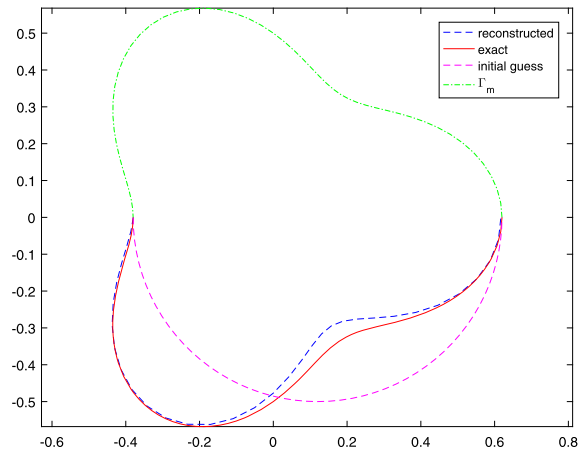

(b) Using noisy data with $\delta=2 \%, 7$ iteration steps

Figure 5 Reconstruction the corrosion boundary of pear with exact data above and with $2 \%$ noisy data below, respectively. We choose Dirichlet data on $\Gamma_{m}$ as $f\left(z_{m}(t)\right)=1+\cos ^{2}(t), t \in[0, \pi]$, the impedance coefficients on boundary $\Gamma_{C}$ are chosen as $\lambda=0.8+\cos ^{4} t$ and $\mu=\frac{1}{1+0.15 \cos 2 t}, t \in[\pi, 2 \pi]$

$f\left(z_{m}(t)\right)=1, t \in[0, \pi]$, and the impedance coefficients on boundary $\Gamma_{c}$ as

$$
\lambda=0.8+\cos ^{4} t \quad \text { and } \quad \mu=\frac{1}{1+0.15 \cos 2 t}, \quad t \in[\pi, 2 \pi] .
$$

Using the exact Cauchy data with 7 iteration steps and the noisy data with $\delta=5 \%$ and 9 iteration steps, we establish the reconstruction of the ellipse in Fig. 1 . To consider the influence of different Dirichlet data to the reconstruction, we choose $f\left(z_{m}(t)\right)=1+\cos ^{2}(t)$, $t \in[0, \pi]$, as Dirichlet data on $\Gamma_{m}$ in Fig. 2, and the generalized impedance coefficients on the boundary $\Gamma_{c}$ are still given in (28). Using the exact Cauchy data with 13 iteration steps and the noisy data with $\delta=3 \%$ and 13 iteration steps, we establish the reconstruction of the ellipse in Fig. 2. We choose $f\left(z_{m}(t)\right)=1+\cos ^{2}(t), t \in[0, \pi]$, as Dirichlet data on $\Gamma_{m}$ and the impedance coefficients on the boundary $\Gamma_{c}$ given as two constants $\lambda=1$ and $\mu=3$ in Fig. 3. Using the exact Cauchy data with 12 iteration steps and the noisy data with $\delta=3 \%$ and 13 iteration steps, we establish the reconstruction of the ellipse in Fig. 3. 


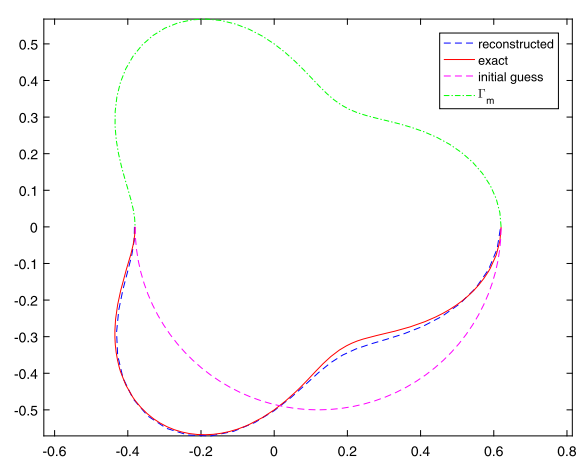

(a) Using exact Cauchy data, 9 iteration steps

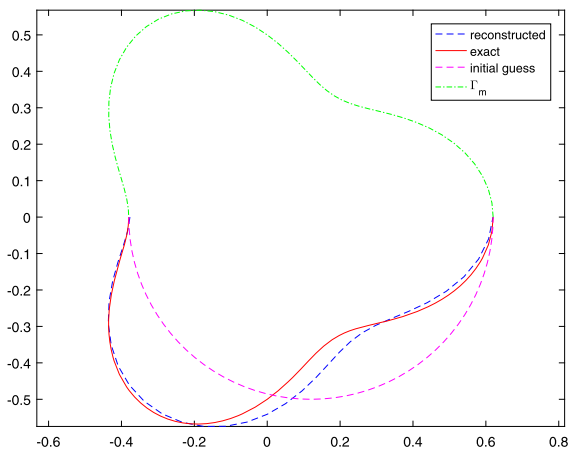

(b) Using noisy data with $\delta=2 \%, 13$ iteration steps

Figure 6 Reconstruction the corrosion boundary of pear with exact data above and with $2 \%$ noisy data below, respectively. We choose Dirichlet data on $\Gamma_{m}$ as $f\left(z_{m}(t)\right)=1+\cos ^{2}(t), t \in[0, \pi]$, the impedance coefficients on boundary $\Gamma_{c}$ are chosen as two constants $\lambda=1$ and $\mu=3$

Example 2 We consider the parametric form of pear-shaped boundary

$$
z_{c}(t)=(0.5+0.12 \cos (3 t))(\cos t, \sin t), \quad t \in[\pi, 2 \pi],
$$

and choose $m=3$ in (24) and the given parametric form of known boundary $\Gamma_{m}$

$$
z_{m}(t)=(0.5+0.12 \cos (3 t))(\cos t, \sin t), \quad t \in[0, \pi] .
$$

The initial value of the boundary $\Gamma_{c}$ is the lower half-circle with center $(0.12,0)$ and radius 0.5 . We choose $f\left(z_{m}(t)\right)=1, t \in[0, \pi]$, as Dirichlet data on $\Gamma_{m}$ in Fig. 4, and the generalized impedance coefficients on the boundary $\Gamma_{c}$ are still given in (28). Using the exact Cauchy data with 7 iteration steps and the noisy data with $\delta=2 \%$ with 9 iteration steps, we establish the reconstruction of the pear-shaped boundary in Fig. 4. To consider the influence of different Dirichlet data to reconstruct the boundary, we choose $f\left(z_{m}(t)\right)=1+\cos ^{2}(t)$, $t \in[0, \pi]$, as Dirichlet data on $\Gamma_{m}$ in Fig. 5, and the coefficients on boundary $\Gamma_{c}$ are still 


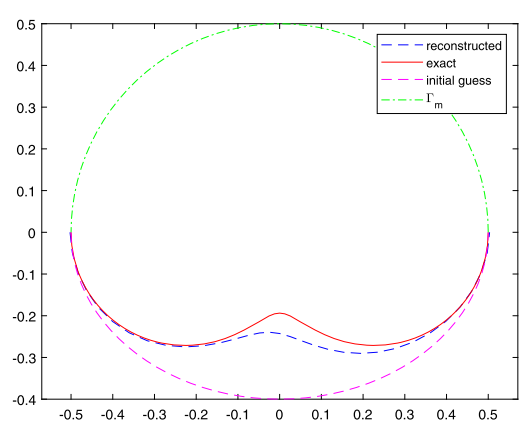

(a) Using exact Cauchy data, 9 iteration steps

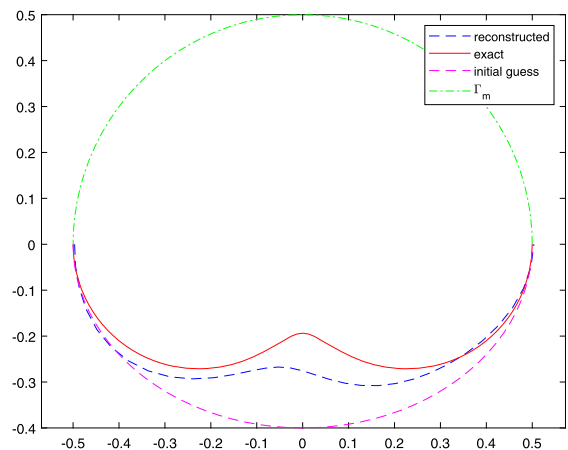

(b) Using noisy data with $\delta=2 \%, 12$ iteration steps

Figure 7 Reconstruction of shape (32) with exact data above and with 2\% noisy data below, respectively. We choose Dirichlet data on $\Gamma_{m}$ as $f\left(z_{m}(t)\right)=1+\cos ^{2}(t), t \in[0, \pi]$, the impedance coefficients on boundary $\Gamma_{c}$ are chosen as $\lambda=\frac{1}{1-0.1 \sin 2 t}$ and $\mu=\frac{1}{1+0.3 \cos t}, t \in[\pi, 2 \pi]$

given in (28). Using the exact Cauchy data with 7 iteration steps and the noisy data with $\delta=2 \%$ and 7 iteration steps, we establish the reconstruction of the pear-shaped boundary in Fig. 5. We choose $f\left(z_{m}(t)\right)=1+\cos ^{2}(t), t \in[0, \pi]$, as Dirichlet data on $\Gamma_{m}$, and the impedance coefficients on the boundary $\Gamma_{c}$ are given as two constants $\lambda=1$ and $\mu=3$ in Fig. 6. Using the exact Cauchy data with 9 iteration steps and the noisy data with $\delta=2 \%$ and 13 iteration steps, we establish the reconstruction of the pear-shaped boundary in Fig. 6. Considering different Dirichlet data and different generalized impedance coefficients on the boundary, we obtain good reconstruction results.

In the previous examples, the boundary $\partial D=\bar{\Gamma}_{m} \cup \bar{\Gamma}_{c}$ of the domain $D$ is smooth. We further consider piecewise smooth boundaries.

Example 3 We consider a piecewise smooth boundary with corners at the intersection points. For the latter, the upper part $\Gamma_{m}$ is a circle given by

$$
z_{m}(t)=0.5(\cos t, \sin t), \quad t \in[0, \pi],
$$




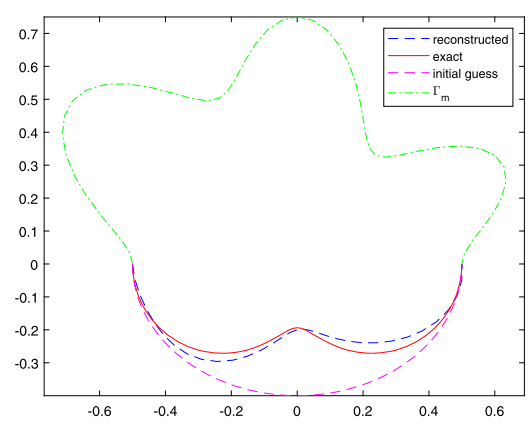

(a) Using exact Cauchy data, 12 iteration steps

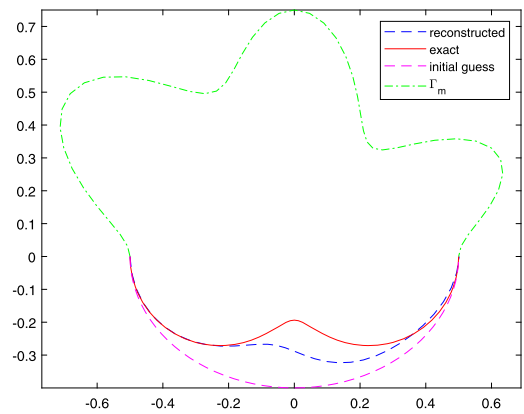

(b) Using noisy data with $\delta=2 \%, 15$ iteration steps

Figure 8 Reconstruction of shape (34) with exact data above and with $2 \%$ noisy data below, respectively. We choose Dirichlet data on $\Gamma_{m}$ as $f\left(z_{m}(t)\right)=1+\cos ^{2}(t), t \in[0, \pi]$, the impedance coefficients on boundary $\Gamma_{c}$ are chosen as $\lambda=\frac{1}{1-0.1 \sin 2 t}$ and $\mu=\frac{1}{1+0.3 \cos t}, t \in[\pi, 2 \pi]$

and the lower part $\Gamma_{c}$ is a peanut-shaped contour given by

$$
z_{c}(t)=\left(0.5 \sqrt{\cos ^{2} t+0.15 \sin ^{2} t}\right)(\cos t, \sin t), \quad t \in[\pi, 2 \pi] .
$$

We choose $m=5$ in (24). The initial value of the boundary $\Gamma_{c}$ is the lower half-ellipse given as $z_{c_{0}}(t)=(0.5 \cos t, 0.4 \sin t), t \in[\pi, 2 \pi]$, with end points coinciding with the end points $z(0)$ and $z(\pi)$ of $\Gamma_{m}$. We choose $f\left(z_{m}(t)\right)=1+\cos ^{2}(t), t \in[0, \pi]$, as Dirichlet data on $\Gamma_{m}$ in Fig. 7, and the generalized impedance coefficients on the boundary $\Gamma_{c}$ are given as $\lambda=\frac{1}{1-0.1 \sin 2 t}$ and $\mu=\frac{1}{1+0.3 \cos t}, t \in[\pi, 2 \pi]$. Using the exact Cauchy data with 9 iteration steps and the noisy data with $\delta=2 \%$ and 12 iteration steps, we establish the reconstruction of the lower part of the peanut-shaped boundary in Fig. 7.

Example 4 We also consider a piecewise smooth boundary with corners at the intersection points. For the latter, the upper part $\Gamma_{m}$ is given by

$$
z_{m}(t)=\left(0.5+0.25 \cos ^{2}(3 t)-0.15 \sin ^{5}(2 t)\right)(\sin t, \cos t), \quad t \in[0, \pi]
$$


and the lower part $\Gamma_{c}$ is a peanut-shaped contour given by

$$
z_{c}(t)=\left(0.5 \sqrt{\cos ^{2} t+0.15 \sin ^{2} t}\right)(\cos t, \sin t), \quad t \in[\pi, 2 \pi] .
$$

We choose $m=7$ in (24). The initial value of the boundary $\Gamma_{c}$ is the lower half-ellipse given as $z_{c_{0}}(t)=(0.5 \cos t, 0.4 \sin t), t \in[\pi, 2 \pi]$, with end points coinciding with the end points $z(0)$ and $z(\pi)$ of $\Gamma_{m}$. We choose $f\left(z_{m}(t)\right)=1+\cos ^{2}(t), t \in[0, \pi]$, as Dirichlet data on $\Gamma_{m}$ in Fig. 8, and the generalized impedance coefficients on the boundary $\Gamma_{c}$ are given as $\lambda=\frac{1}{1-0.1 \sin 2 t}$ and $\mu=\frac{1}{1+0.3 \cos t}, t \in[\pi, 2 \pi]$. Using the exact Cauchy data with 12 iteration steps and the noisy data with $\delta=2 \%$ and 15 iteration steps, we establish the reconstruction of the lower part of the peanut-shaped boundary in Fig. 8.

\section{Concluding remarks}

The numerical examples indicate that our proposed Newton method provides good stable reconstructions of the corrosion boundary. More analysis is needed to reconstruct two generalized impedance coefficients on the corrosion boundary, and also more research is needed to consider the uniqueness of the inverse Wentzell-type GIBC problem.

\section{Acknowledgements}

The authors would like to thank the anonymous referees for very helpful suggestions and comments, which led to improvement of our original manuscript.

\section{Funding}

The research of Y.Q. Hu is supported by PhD research startup foundation of Jinling Institute of Technology

(No. jit-b-201524) and the Science Foundation of Jinling Institute of Technology (No. Jit-fhxm-201809).

Availability of data and materials

Contact the authors for data requests.

Competing interests

The authors declare that they have no competing interests.

Authors' contributions

All authors have given approval to the final version of the manuscript.

\section{Author details}

${ }^{1}$ School of Science, Jinling Institute of Technology, Hongjing Road 99, Nanjing, 211169, P.R. China. ${ }^{2}$ School of Mathematical Science, Huaiyin Normal University, Changjiang Road 111, Huaian, 223300, P.R. China. ${ }^{3}$ School of Mathematics and Statistics, Taishan University, Dongyue Street 525, Taian, 271000, P.R. China.

\section{Publisher's Note}

Springer Nature remains neutral with regard to jurisdictional claims in published maps and institutional affiliations.

Received: 4 September 2019 Accepted: 27 July 2020 Published online: 05 August 2020

\section{References}

1. Bacchelli, V:: Uniqueness for the determination of unknown boundary and impedance with the homogeneous Robin condition. Inverse Probl. 25(1), 1 (2008)

2. Cakoni, F., Colton, D.: The determination of the surface impedance of a partially coated obstacle from far field data. SIAM J. Appl. Math. 64(2), 709-723 (2003)

3. Cakoni, F., Colton, D., Monk, P.: The direct and inverse scattering problems for partially coated obstacles. Inverse Probl. 17(6), 1997-2015 (2002)

4. Cakoni, F., Colton, D., Monk, P.: The determination of the surface conductivity of a partially coated dielectric. SIAM J. Appl. Math. 65(3), 767-789 (2005)

5. Cakoni, F., Colton, D., Monk, P.: The inverse electromagnetic scattering problem for a partially coated dielectric. J. Comput. Appl. Math. 204(2), 256-267 (2007)

6. Cakoni, F., Colton, D., Monk, P.: The determination of boundary coefficients from far field measurements. J. Integral Equ. Appl. 22(2), 167-191 (2010)

7. Cakoni, F., Hu, Y.Q., Kress, R.: Simultaneous reconstruction of shape and generalized impedance functions in electrostatic imaging. Inverse Probl. 30, 10 (2014) 
8. Cakoni, F., Haddar, H., Meng, S.: Boundary integral equations for the transmission eigenvalue problem for Maxwell equations. J. Integral Equ. Appl. 27(3), 787-814 (2015)

9. Cakoni, F., Ivanyshyn, O., Kress, R., Le Louer, F.: A boundary integral equations for the transmission eigenvalue problem for Maxwell equations. Math. Methods Appl. Sci. 41(4), 1316-1330 (2018)

10. Cakoni, F., Kress, R.: Integral equations for inverse problems in corrosion detection from partial Cauchy data. Inverse Probl. Imaging 1(2), 229-245 (2007)

11. Cakoni, F., Kress, R.: Integral equation methods for the inverse obstacle problem with generalized impedance boundary condition. Inverse Probl. 29(1), 129-143 (2013)

12. Cakoni, F., Kress, R.: A boundary integral equation method for the transmission eigenvalue problem. Appl. Anal. 96, 23-38 (2017)

13. Cakoni, F., Kress, R., Schuft, C.: Integral equations for shape and impedance reconstruction in corrosion detection. Inverse Probl. 26, 9 (2010)

14. Cakoni, F., Kress, R., Schuft, C.: Simultaneous reconstruction of shape and impedance in corrosion detection. Methods Appl. Anal. 17(4), 331-462 (2010)

15. Cao, P., Wu, J.H., Chen, X.: An approach to investigate the multiple-scattering problems based on the singularity expansion method. J. Appl. Phys. 123, 13 (2018)

16. Colton, D., Kress, R.: Inverse Acoustic and Electromagnetic Scattering Theory. Applied Mathematical Sciences. Springer, New York (2019)

17. Elliott, D.: Sigmoidal transformations and the trapezoidal rule. J. Aust. Math. Soc. Ser. B, Appl. Math 40,77-137 (1998)

18. Elschner, J., Graham, l.: An optimal order collocation method for first kind boundary integral equations on polygons. Numer. Math. 70(1), 1-31 (1995)

19. Gillman, A.: An integral equation technique for scattering problems with mixed boundary conditions. Adv. Comput. Math. 43(2), 351-364 (2016)

20. Haddar, H., Joly, P., Nguyen, H.: Generalized impedance boundary conditions for scattering problems from strongly absorbing obstacles: the case of Maxwell's equations. Math. Models Methods Appl. Sci. 18(10), 1787-1827 (2008)

21. Ivanyshyn, O., Kress, R.: Nonlinear integral equations for solving inverse boundary value problems for inclusions and cracks. J. Integral Equ. Appl. 18, 13-38 (2006)

22. Ivanyshyn, O., Kress, R.: Nonlinear integral equations in inverse obstacle scattering. In: Mathematical Methods in Scattering Theory and Biomedical Engineering, pp. 39-50 (2006)

23. Kress, R.: Linear Integral Equations. Springer, Berlin (1989)

24. Kress, R., Rundell, W.: Nonlinear integral equations and the iterative solution for an inverse boundary value problem. Inverse Probl. 21(4), 1207-1223 (2005)

25. Liu, J.J., Nakamura, G., Sini, M.: Reconstruction of the shape and surface impedance for acoustic scattering data for an arbitrary cylinder. SIAM J. Appl. Math. 67(4), 1124-1146 (2007)

26. Potthast, R.: Fréchet differentiability of boundary integral operators in inverse acoustic scattering. Inverse Probl. 10(2), 431-447 (1994)

27. Qin, H.H., Cakoni, F.: Nonlinear integral equations for shape reconstruction in the inverse interior scattering problem. Inverse Probl. 27, 3 (2011).

28. Rundell, W.: Recovering an obstacle and its impedance from Cauchy data. Inverse Probl. 24, 4 (2008)

29. Wang, Y., Ma, F., Zheng, E.: A discontinuous Galerkin method for acoustic scattering problem with DtN boundary condition. Appl. Anal. 97(6), 938-961 (2017)

\section{Submit your manuscript to a SpringerOpen ${ }^{\circ}$ journal and benefit from:}

- Convenient online submission

- Rigorous peer review

- Open access: articles freely available online

- High visibility within the field

- Retaining the copyright to your article

Submit your next manuscript at $\boldsymbol{~ s p r i n g e r o p e n . c o m ~}$ 